\title{
Adult patent ductus arteriosus
}

\section{An unusual cause of heart failure in an octogenarian female}

\author{
Silvana Mueller · Fabian Plank · Katrin Klimes · Gudrun Feuchtner · Johannes Mair
}

Received: 4 May 2016 / Accepted: 18 July 2016 / Published online: 26 August 2016

(C) The Author(s) 2016. This article is available at SpringerLink with Open Access.

\begin{abstract}
Summary Patent ductus arteriosus (PDA) is the third most common congenital abnormality in which the arterial duct, which normally closes spontaneously after birth within 24-48 h in full-term infants, remains permanently open. Breathlessness is very common in elderly patients and can be caused by several comorbidities, mostly cardiac and pulmonary diseases. PDA as a cause of heart failure in this patient population is very rare and diagnosis depends on high clinical awareness. Here we present a case diagnosed with multimodality imaging including 3-dimensional (3D) transthoracic and transesophageal echocardiography and 3D-volume rendering technique (VRT) computed tomography.
\end{abstract}

Keywords Patent ductus arteriosus - Heart failure . Dyspnea $\cdot$ Octogenarians

\section{Introduction}

A patent ductus arteriosus (PDA) is the third most common congenital abnormality in which the arterial duct, which normally closes spontaneously after birth within 24-48 $\mathrm{h}$ in full-term infants, remains permanently open [1]. Breathlessness is very com-

\section{K. Klimes, MD}

Department of Internal Medicine III - Cardiology and Angiology, Department of Pediatric Cardiology, Innsbruck Medical University, 6020 Innsbruck, Austria

\section{G. Feuchtner, MD}

Department of Radiology, Innsbruck Medical University, 6020 Innsbruck, Austria

\section{J. Mair, MD ( $\varangle)$}

Department of Internal Medicine III - Cardiology

and Angiology, Innsbruck Medical University,

Anichstrasse 35, 6020 Innsbruck, Austria

Johannes.Mair@i-med.ac.at mon in elderly patients and can be caused by several comorbidities, mostly cardiac and pulmonary diseases. The PDA as a cause of heart failure in this patient population is very rare and diagnosis depends on high clinical awareness. In this article we present a case diagnosed with multimodality imaging including 3-dimensional (3D) transthoracic and transesophageal echocardiography and 3D-volume rendering technique (VRT) computed tomography (CT).

\section{Case report}

An 84-year-old female was referred for cardiac catheterization for evaluation of a suspected significant coronary artery disease and mitral valve regurgitation. The patient complained of worsening of dyspnea symptoms during exercise, New York Heart Association (NYHA) class II-III for several months without angina. The resting electrocardiogram (ECG) showed atrial fibrillation with a heart rate of $90 / \mathrm{min}$ (see supplemental figure 1). The N-terminal pro B-type natriuretic peptide (NT-proBNP) level was moderately elevated (2274 ng/l) with normal renal and thyroid function and borderline anemia (hemoglobin $116 \mathrm{~g} / \mathrm{l}$ ). Transthoracic and transesophageal 3D echocardiography (see Fig. 1, upper panels, supplemental video 1) revealed a rare cause of heart failure in octogenarians, i. e. a partially calcified but patent ductus arteriosus $6 \mathrm{~mm}$ in diameter, which was confirmed by 3DVRT computed tomography (see Fig. 1, lower panels, supplemental video 2). All 4 heart chambers were dilated (echocardiography: left ventricular enddiastolic diameter $67 \mathrm{~mm}$, right ventricle $35 \mathrm{~mm}$, left atrium $59 \mathrm{~mm}$ and right atrium $48 \mathrm{~mm}$ ), the pulmonary arteries (right $40 \mathrm{~mm}$ and left $30 \mathrm{~mm}$ ) and the pulmonary trunk $(55 \mathrm{~mm})$ were severely dilated. Left ventricular systolic function was slightly 
Fig. 1 Multimodality imaging of patent partially calcified ductus arteriosus in an octogenarian female. Upper panels show images of transesophageal and 3-D transthoraracic echocardiography, lower panels images of computed and 3D-VRT computed tomography. The diameter of the patent ductus arteriosus (arrows) was $6 \mathrm{~mm}$, calcification is marked with asterisks $\left.{ }^{(*}\right)$. $A A$ ascending aorta, $A D$ descending aorta, $T P$ pulmonary trunk
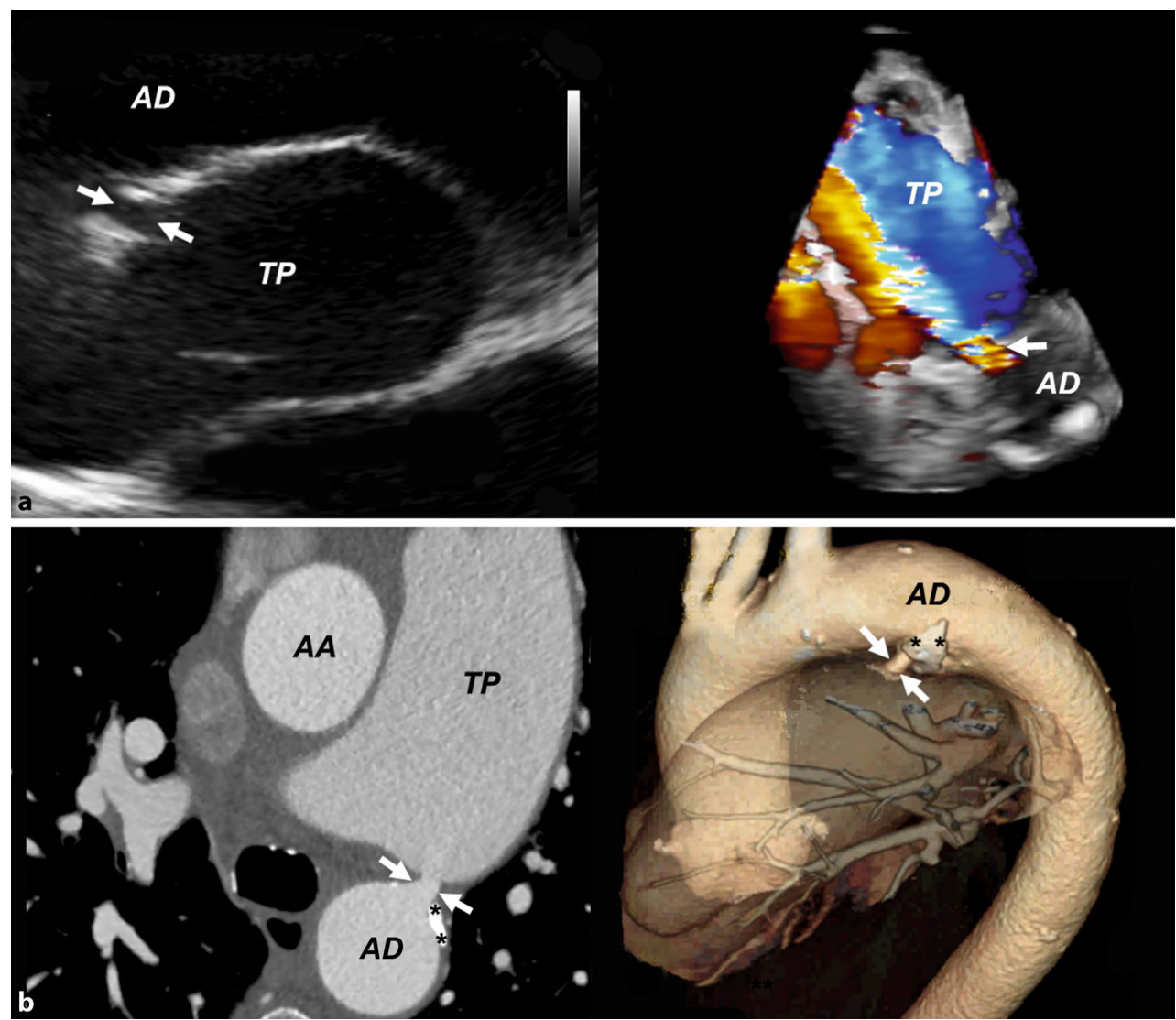

decreased (left ventricular ejection fraction $45 \%$ ) and the right ventricular systolic function was normal. There was only mild to moderate mitral valve regurgitation and moderate pulmonary valve and tricuspid valve regurgitation. Cardiac catheterization revealed a significant left to right shunt of 0.56 (arterial, right atrial and pulmonary artery oxygen saturation was and $93 \%, 27 \%$ and $65 \%$, respectively) and severe pulmonary arterial hypertension (systolic $62 \mathrm{~mm} \mathrm{Hg}$ and diastolic $28 \mathrm{~mm} \mathrm{Hg}$, mean $42 \mathrm{~mm} \mathrm{Hg}$, the pulmonary artery occlusion pressure was at maximum $14 \mathrm{~mm} \mathrm{Hg}$, mean $10 \mathrm{~mm} \mathrm{Hg}$; transpulmonary gradient $32 \mathrm{mmHg}$, diastolic pulmonary pressure gradient $18 \mathrm{mmHg}$. Significant coronary artery disease was ruled out by coronary angiography.

The subsequent detailed medical history revealed that the patient was first informed of the presence of a cardiac murmur at the age of 23 years and a holosystolic 3/6 systolic murmur (best heard right second intercostal space) could be heard at presentation to our department. At this young age the patient was asymptomatic but over the subsequent decades the substantial left to right shunt led to left ventricular dysfunction and severe pulmonary arterial hypertension.

\section{Discussion}

It is very rare to diagnose a PDA in an octogenarian and the diagnosis requires a high clinical awareness as this congenital heart disease is uncommon in this patient population. The oldest published case was 91 years old [2] and patients can only occasionally survive over 50 years without an intervention [1-6] but our report confirms that PDA can be compatible with normal life expectancy. Systolic murmurs are unspecific as are the transthoracic echocardiographic signs as long as the PDA is not easily visible; however, a retrograde jet flow at the main pulmonary artery should raise the suspicion of PDA. In case of doubt in echocardiography, computed tomography enables definitive diagnosis.

Published data on the management of PDA in octogenarians is very limited $[5,6]$. The relationship between the discovered PDA and the individual symptoms has to be assessed(incidental finding or relevant pathology?). In our case the significant left to right shunt is a plausible explanation for the development of left ventricular dysfunction, severe pulmonary hypertension and atrial fibrillation. A case of percutaneous occlusion of PDA with an Amplatzer duct occluder device in an 80-year-old man with moderate pulmonary hypertension has been reported [6]; however, transcatheter PDA closure may not be safe in patients with severe pulmonary hypertension and the 
PDA calcification seen in our patient even increases the interventional risk. Therefore, we started specific vasodilatory medication treatment of pulmonary arterial hypertension with an endothelin antagonist (macitentan $10 \mathrm{mg}$ per day) during the hospital stay to lower the pulmonary pressure. At the 1-year followup visit the patient presented with a good clinical condition, improved heart failure symptoms (NYHA II) and a significant decrease in NT-proBNP (808 ng/l). The transcutaneously measured oxygen saturation in the upper and lower limbs were $99 \%$ each at rest and post-ductal $92 \%$ in the lower limbs after a 6 min walking test (walking distance $420 \mathrm{~m}$ ).

\section{Caption electronic supplementary material}

- Fig. 1: Resting electrocardiogram at first presentation

- Supplemental video 1: Three-dimensional echocardiography of patent ductus arteriosus

- Supplemental video 2: 3D-VRT computed tomography of patent ductus arteriosus

Open access funding provided by University of Innsbruck and Medical University of Innsbruck.

Conflict of interest S. Mueller, F. Plank, K. Klimes, G. Feuchtner, and J. Mair declare that they have no competing interests.
Open Access This article is distributed under the terms of the Creative Commons Attribution 4.0 International License (http://creativecommons.org/licenses/by/4.0/), which permits unrestricted use, distribution, and reproduction in any medium, provided you give appropriate credit to the original author(s) and the source, provide a link to the Creative Commons license, and indicate if changes were made.

\section{References}

1. Schneider DJ, Moore JW. Patent ductus arteriosus. Circulation. 2006;114:1873-82.

2. Satoh T, Yanagitani Y, Okano Y. Patent ductus arteriosus with combined valvular disease at age 91. Intern Med. 1997;36:340-4.

3. Salvana EMT. Patent ductus arteriosus in an elderly man. NewEngl J Med. 2006;354:2484.

4. Marek T, Zelizko M, Kautzner J. Real-time 3-dimensional transesophageal echocardiography imaging-adult patent ductus arteriosus before and after transcatheter closure. Circulation. 2009;120:e92-3.

5. Boyalla V, Putzu P, Dierckx R, Clark AL, Pellicori P. Patent ductus arteriosus in older adults: incidential finding or relevant pathology? J Am Geriatr Soc. 2015;63:409-11.

6. Lee HY, Her SH, ParkWM, Choi MS, Cho JS, Kim CJ, KangHS, Choi YA, Ju IN, Kim SA, Lee JH, Kwon JB, Park K. A case of patent ductus arteriosus with congestive heart failure in a 80-year old man. Korean Circ J. 2012;42:849-52. 